\title{
A STUDY OF THE GALACTIC CENTER REGIONS USING THE IMPROVED DATA OF THE MID-INFRARED ALL-SKY SURVEY
}

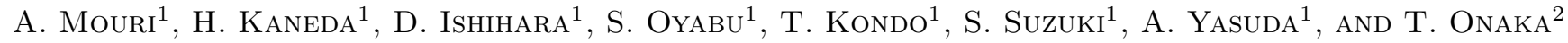 \\ ${ }^{1}$ Graduate School of Science, Nagoya University, Furo-cho, Chikusa-ku, Nagoya, Aichi, 464-8602, Japan \\ ${ }^{2}$ Graduate School of Science, University of Tokyo, 7-3-1, Hongo, Bunkyo-ku, Tokyo, 113-0033, Japan \\ E-mail: ishihara@u.phys.nagoya-u.ac.jp \\ (Received June 30, 2012; Accepted August 20, 2012)
}

\begin{abstract}
Among the AKARI all-sky survey data, the $9 \mu \mathrm{m}$ diffuse map is crucial to study the polycyclic aromatic hydrocarbon (PAH) emission features on large spatial scales, while the $18 \mu \mathrm{m}$ map is useful to trace hot dust emission. To utilize these advantages, we have improved the AKARI mid-infrared (MIR) all-sky survey diffuse maps. For example, we have established special methods to remove the effects of the ionizing radiation in the South Atlantic Anomaly (SAA) and of the scattered light from the moon. Using improved diffuse map data, we study the properties of PAHs and dust in the Galactic center region associated with high-energy phenomena.
\end{abstract}

Key words: infrared; ISM: methods; data analysis: surveys

\section{INTRODUCTION}

The AKARI $9 \mu \mathrm{m}$ all-sky diffuse map traces the interstellar PAH emission features on large spatial scales, while the $18 \mu \mathrm{m}$ map is useful to trace hot dust emission. PAHs are easily destroyed in harsh environments (e.g. shock region, hot plasma), while large grains survive for a relatively long time, heating up to higher temperatures. Using the different lifetimes between PAHs and large grains, we can investigate their processing in the harsh interstellar environment.

To enable these studies, we have improved the AKARI MIR all-sky survey diffuse maps by adding special analysis. Details of the survey and the data processing are given in Ishihara et al. $(2006,2010)$. We focus on two newly-developed special methods in the data processing: the methods to remove the effects of the ionizing radiation in the SAA and of the scattered light from the moon.

\section{NEW ANALYSIS FOR DIFFUSE MAP CON- STRUCTION}

2.1. Correction of Ionizing Radiation Effects

The data taken in the SAA suffer from systematic changes in the output level, even after the spike noise, which is the direct effect of the ionizing radiation, is removed. Because AKARI passes the SAA four times among 14 orbits per day, this effect is significant. We have investigated the amount and the mechanism of this effect and established a method to correct them (Mouri et al., 2010). Figure 1 demonstrates an example of the results after the correction of this effect.

\subsection{Removal of Scattered Light of the Moon}

We evaluate the intensity and the spatial distribution of the scattered light of the moon by constructing the scattered light pattern image in the moon-centered detector-array coordinates. The scattered light pattern shows wider distribution than expected, asymmetry to the in-scan axis, and three pairs of arc structures originating in the spiders of the telescope. We further evaluate the stability of the pattern among seasons, and 


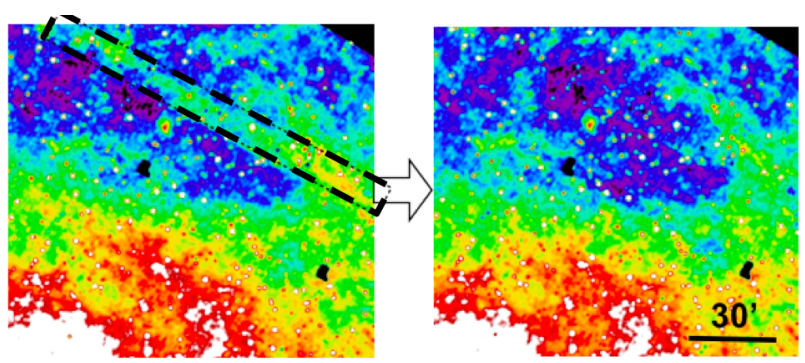

Fig. 1. Before (left) and after (right) the correction of the ionizing radiation effects in the SAA. This example is a $3 \times 3$ deg $^{2}$ image near the Galactic center. The faint diffuse structure indicated by the dashed line on the left image is found to be artifacts.

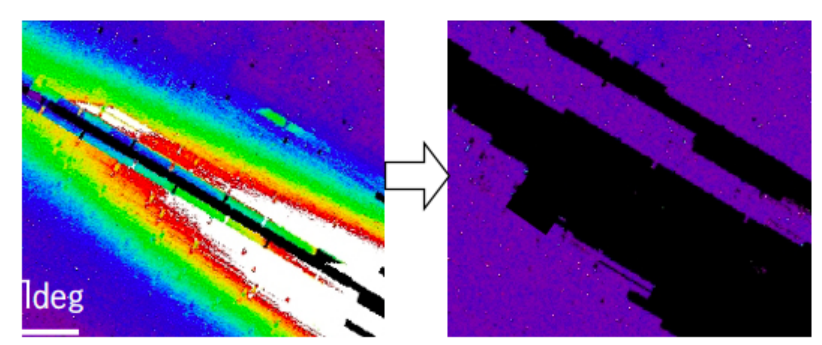

Fig. 2. Before (left) and after (right) the correction of the scattered light of the moon. This example is a $5 \times 5$ $\operatorname{deg}^{2}$ image covered in one season.

investigate the reason for the asymmetry of the pattern. Using this pattern as a template, we remove the scattered light component from the maps. Figure 2 demonstrates an example of the results after the successful removal.

\section{PAHS IN THE HARSH ENVIRONMENT}

Using the improved diffuse map data, we study the properties of PAHs and dust in the Galactic center region associated with high-energy phenomena.

\subsection{Galactic Molecular Loop}

For the molecular loops located at around $(l, b)=\left(-3^{\circ}\right.$, $\left.+1^{\circ}\right)$, which were discovered by Fukui et al. (2006) in the ${ }^{12} \mathrm{CO}(\mathrm{J}=1-0)$ emission, we find that the ratios of the $9 \mu \mathrm{m}$ to the $100 \mu \mathrm{m}$ emission are suppressed at their foot points, indicating that the PAHs are selectively destroyed due to the shock excited by the collision of disk gas with the gas sliding down along the loops (Kaneda et al., 2012).

\subsection{Bubble in the Galactic Center}

For the bubble structure centered at around $(l$, $b)=\left(0.14^{\circ},-0.12^{\circ}\right)$ near the Quintuplet cluster, we find that the PAH emission is faint while the dust temperature is enhanced, supporting the idea that the structure is excited by shock winds from Wolf-Rayet stars in the Quintuplet Cluster.

\section{SUMMARY}

We have established special methods to remove the effects of the ionizing radiation in the SAA and of the scattered light from the moon to improve the AKARI/MIR all-sky diffuse maps. Using the improved diffuse maps, we have studied the properties of PAHs and dust in the Galactic center region associated with high-energy phenomena. We have established the method to probe interstellar phenomena using the difference in the lifetime between PAHs and large grains in the harsh environment.

\section{ACKNOWLEDGEMENTS}

This work was supported by Grants-in-Aid for Scientific Research (24740122) from the Japan Society for the Promotion of Science (JSPS), and the Nagoya University Global Center of Excellence Program, "Quest for Fundamental Principles in the Universe" (QFPU) from JSPS and the Ministry of Education, Culture, Sports, Science and Technology of Japan.

\section{REFERENCES}

Fukui, Y., et al., 2006, Effects of High-Energy Ionizing Particles on the Si:As Mid-Infrared Detector Array on Board the AKARI Satellite, PASP, 123, 561

Ishihara, D., et al., 2006, Mid-Infrared All-Sky Survey with the Infrared Camera (IRC) on Board the ASTRO-F Satellite, PASP, 118, 324

Ishihara, D., et al., 2010, The AKARI/IRC MidInfrared All-Sky Survey, A\&A, 514, 1

Kaneda, H., et al., 2012, Processing of Polycyclic Aromatic Hydrocarbons in Molecular-Loop Regions near the Galactic Center Revealed by AKARI, PASJ, 64, 25

Mouri, A., et al., 2011, Effects of High-Energy Ionizing Particles on the Si:As Mid-Infrared Detector Array on Board the AKARI Satellite, PASP, 123, 561 